\title{
Le procès Corday : retour aux sources
}

\section{Guillaume Mazeau}

\section{(2) OpenEdition}

\section{Journals}

\section{Édition électronique}

URL : https://journals.openedition.org/ahrf/9812

DOI : 10.4000/ahrf.9812

ISSN : 1952-403X

Éditeur :

Armand Colin, Société des études robespierristes

Édition imprimée

Date de publication : 15 mars 2006

ISSN : 0003-4436

Référence électronique

Guillaume Mazeau, "Le procès Corday : retour aux sources », Annales historiques de la Révolution française [En ligne], 343 | janvier-mars 2006, mis en ligne le 01 mars 2009, consulté le 23 avril 2022 URL : http://journals.openedition.org/ahrf/9812 ; DOI : https://doi.org/10.4000/ahrf.9812

Ce document a été généré automatiquement le 23 avril 2022.

Tous droits réservés 


\title{
Le procès Corday : retour aux sources
}

\author{
Guillaume Mazeau
}

1 Pendant deux siècles, l'assassinat de Marat fut un des piliers d'une mémoire bipolaire opposant «la Révolution» (Marat) à « la contre-révolution» (Corday). Présentant Charlotte Corday en ennemie de la République ou au contraire en martyre de la Terreur, cette historiographie, fondée sur des réécritures, a produit des récits stéréotypés et téléologiques qui, parce qu'ils ne correspondent plus au contexte idéologique contemporain, s'épuisent lentement. Les grands paradigmes ont en effet entraîné dans leur chute leurs points d'appui historiographiques, et les historiens se sont détournés de ces vestiges, désormais dépourvus de légitimité idéologique. Sans remous, le 13 juillet 1793 s'efface des chronologies « officielles » de la Révolution.

2 Ce silence ne peut pourtant satisfaire, lorsqu'on le compare au séisme que l'événement a suscité en $1793^{1}$, et quand on réalise les enjeux qu'il soulève aujourd'hui. En ce début de $\mathrm{XXI}^{\mathrm{e}}$ siècle, s'interroger sur la manière dont une inconnue a décidé de peser sur l'histoire en provoquant une crise panique au cœur du pouvoir par un attentat que l'on ne peut qualifier que de "terroriste", ne doit rien au hasard. L'historicité de cette question incite à se méfier des interprétations partisanes, provoquées par l'émotion et les conflits politiques immédiats. Elle oblige aussi à revenir sur l'importance de l'événement qui fait surgir de nouvelles pratiques et images sociales ${ }^{2}$. Elle contraint enfin les universitaires à occuper le terrain et à contester l'image actuellement dominante de Corday, issue de la mémoire contre-révolutionnaire, reprise ensuite par l'extrême droite, et majoritairement relayée par les biographes ${ }^{3}$ : celle d'une martyre inspirée par la religion dont le sacrifice aurait purgé la France de l'infâme Marat.

3 Un retour aux sources (le dossier du procès, mais aussi les brochures et les journaux) a permis de séparer au maximum les faits de leurs interprétations postérieures, sans ignorer le caractère aporétique d'une telle distinction. Comprise strictement, celle-ci reviendrait à cliver artificiellement l'histoire et la mémoire, les pratiques et les représentations, réduisant l'entreprise historiographique à un travail d'érudition sans lien réel avec ce qui s'est passé, voire à une volonté idéologique de légitimer ou 
délégitimer le travail des historiens ${ }^{4}$. Cette vision nous semble excessive. "Il est important de suivre les étapes des interprétations successives recomposant la trame des faits, pour en tirer des leçons inédites, et mettre en exergue les solutions de continuité qui furent à l'œuvre ${ }^{5}$ : envisagé de la sorte, le travail historiographique n'est pas une simple histoire des représentations à visée démythificatrice, mais bien la reconstitution des faits et de leurs conséquences dans l'histoire ${ }^{6}$ : en retraçant les généalogies mémorielles, il démontre la continuité entre les événements, eux-mêmes impliqués dans de nombreux systèmes de représentations, et les nombreuses images et pratiques sociales qu'ils ont engendrées. Cette approche restitue à l'événement une complexité et un intérêt heuristique finalement plus crédibles que l'image d'Épinal qui nous en parvient souvent. L'abandon des visions globales se traduira ici par une certaine fragmentation du récit : trois niveaux de relectures seront donc proposés.

Une relecture politique et judiciaire

L'été 1793 concentre toutes les peurs : la guerre et les conflits intérieurs font craindre un complot contre la République, alors que les Parisiens s'apprêtent à célébrer la nouvelle Constitution le 14 juillet. C'est pourquoi personne ne s'étonne de l'origine normande de Corday, qui donne (comme la Vendée depuis mars) un visage à l'Ouest contre-révolutionnaire tout entier. Les protagonistes du procès partagent, même inconsciemment, cette conviction. Carlo Ginzburg a ainsi montré que dans le procès Sofri, la pression du crime organisé dans l'Italie des années 1980-1990, avait pesé lourd sur les présupposés du procureur et du juge ${ }^{7}$. De même, le procès Corday a été entièrement orienté par le besoin de répondre aux menaces qui pèsent alors sur le pouvoir. La Convention l'affirme dès le 14 juillet: il s'agit d'organiser "le procès de l'assassin de Marat et ses complices»: Corday ne doit pas, ne peut pas avoir agi seule ${ }^{8}$. L'interrogatoire mené à la Conciergerie par Montané, président du Tribunal criminel révolutionnaire, en est la traduction. Ses questions visent à satisfaire la demande de vengeance en démantelant le réseau dont Corday serait l'agent secret. L'attentat est donc perçu à travers une peur à laquelle on donne une explication rassurante: le complot contre-révolutionnaire. Cette vision binaire a influencé l'historiographie pour deux siècles.

5 Dans une atmosphère de fin du monde où l'on redoute d'autres attentats, les rumeurs d'une vengeance populaire se propagent vite et inquiètent Pache, le maire de Paris ${ }^{9}$. Les souvenirs de septembre 1792 exigent une réponse politique rapide: dès le 13, l'ordre d'arrestation est donné à la fois par le Comité de Sûreté générale et la Commune, puis le mandat d'arrêt est rédigé séance tenante. L'hôtel où loge Corday est perquisitionné trois heures après la mort de Marat, pendant le procès-verbal de flagrant délit ${ }^{10}$. Dans la nuit, Corday est mise au secret et gardée à vue à l'Abbaye ${ }^{11}$ alors qu'au-dehors, la confusion règne : le personnel de la section du Théatre-Français, dont le commissaire Guellard a dressé le procès-verbal, doit faire face à un afflux constant de délégués des différentes institutions et assemblées, pressées de recueillir des informations sur l'attentat. Le dossier des pièces présentées lors du procès est alors élaboré comme une vraie arme de frappe. Juste après la mort de Marat, le département de police de la Commune réclame le procès-verbal, l'interrogatoire et une partie des pièces à conviction rassemblées dans la soirée par Guellard. Dès 8 heures le lendemain matin, ces pièces et une copie du procès-verbal de flagrant délit sont dépêchées au bureau du Comité de Sûreté générale ${ }^{12}$. Toute une machine administrative se met en marche : les commis greffiers s'activent, améliorant la circulation des pièces, pressés par les demandes répétées des différentes institutions (le département de police de Paris, puis 
le Tribunal révolutionnaire, mais aussi la mairie de Paris et le Comité de Sûreté générale réclament des copies) ${ }^{13}$. Dès le ${ }^{14}$ juillet, l'accusateur public Fouquier-Tinville, à peine chargé de l'affaire, commence à collecter les pièces dans l'intention d'organiser le procès dès le lendemain14. Le matin même, il est convoqué avec le maire de Paris chez le ministre de la Justice pour définir une ligne officielle. Deux jours plus tard, on mobilise les juges du Tribunal révolutionnaire pour recueillir la dizaine de témoignages à charge ${ }^{15}$; le procès et l'exécution ont lieu le $17^{16}$. On a donc bien exploité les possibilités du décret du 9 mars 1793, qui rendait les jugements exécutables dans les 24 heures et sans aucun recours, mais non sans une certaine improvisation.

6 Parallèlement, la maîtrise politique de l'événement passe par l'élaboration d'un vrai régime de la (fausse) preuve. L'activité déployée par Maure et Chabot, membres du Comité de Sûreté générale, vise à démontrer la complicité des Girondins en falsifiant les pièces à conviction. Le 14 juillet, Lauze Duperret est accusé d'avoir vu Corday juste avant l'attentat; il possède d'ailleurs sa carte de visite. Selon Maure, celle-ci prouve que c'est bien lui qui a envoyé Corday chez Marat: le papier, l'encre et l'écriture sont en effet identiques à ceux qui ont été utilisés par Corday le soir de l'attentat pour se rappeler son adresse ${ }^{17}$. Or un simple examen des archives suffit à le démentir : le papier est différent, le crayon à papier a remplacé l'encre et l'écriture irrégulière accréditent la version de Corday, selon qui c'est un cocher de fiacre qui lui a donné l'information ${ }^{18}$. D'autre part, Chabot, qui a assisté à la saisie des pièces à conviction, a probablement gardé la première lettre adressée à Marat: le résumé qu'il en donne le 14 juillet à la Convention est plus défavorable à Corday que l'original. La seconde lettre, dans laquelle Corday fait appel à la compassion de Marat, a quant à elle servi une célèbre opération de propagande : David la peint dans la main du cadavre pour suggérer la perfidie de Corday qui, en réalité, ne la lui a jamais donnée. Le procès est donc une construction politique ad hoc: alors que les dossiers d'instruction et d'audience doivent normalement être réunis après le jugement, Fouquier-Tinville garde le premier afin de retenir toutes les pièces pouvant servir au procès des complices présumés de Corday, Duperret et Fauchet ${ }^{19}$. Des faux-témoins sont produits : ainsi, un employé à la mairie jure avoir vu Corday tenter de s'introduire dans le bureau de Pache ${ }^{20}$. Cette visite (inventée lors de la réunion du 14 juillet entre Pache, Fouquier-Tinville et le ministre de la justice ?) sert efficacement les Montagnards, qui parient que le dévoilement d'un vaste complot provoquera un mouvement d'adhésion au pouvoir en place. Le procès introduit en tout cas de nouvelles pratiques judiciaires, oblige les institutions à coopérer et pousse les individus à prendre parti. C'est donc une étape dans la construction de rouages politiques et judiciaires d'exception: trois jours plus tard, Fouquier-Tinville, débordé, réclame à la Convention un quatrième juge pour le tribunal $^{21}$.

7 Mais on navigue aussi à vue. Les approximations sont nombreuses dans la collecte des pièces, qui passent entre plusieurs mains avant d'être expédiées à un Fouquier-Tinville las de sonner à toutes les portes. Dès le 14 juillet à l'aube, il presse la Commune de Paris de lui donner le procès-verbal de flagrant délit. Le soir même, il ne l'a toujours pas reçu. Que s'est-il passé ? Il a d'abord fallu que la Commune envoie chercher les pièces à la section du Théâtre-Français, dont le commissaire Guellard avait dressé le procèsverbal la veille au soir. Le transfert a pris une heure, tout au plus ${ }^{22}$. Mais, au lieu d'être expédiées au Tribunal révolutionnaire, elles ont aussitôt été confiées au coursier du Comité de Sûreté générale, probablement mandaté par Chabot afin de préparer son rapport à la Convention, qui se réunissait à peine deux heures plus tard. Les 
empiètements entre les institutions, notamment le Comité de Sûreté générale et le Tribunal révolutionnaire, ont donc « ralenti » la procédure. Le 15 juillet vers 21 heures, l'accusateur public détient presque toutes les pièces dont il a besoin. Ou presque : le lendemain matin, travaillant au tribunal depuis 7 heures, Montané et lui ne peuvent commencer la procédure et pressent la Convention d'intervenir auprès du comité pour qu'il leur ouvre les portes de son bureau ${ }^{23}$. Ceci fait, tout s'accélère alors. Mais dans la mêlée, certaines pièces se sont "égarées": c'est le cas de l'Adresse aux Français, pamphlet écrit par Corday la veille de l'attentat. Fouquier ne l'a pas utilisée contre elle pendant le procès, ce qui a détourné l'accusation, puis l'historiographie, des vrais mobiles politiques de l'attentat contre Marat.

Même pour la censure, on tergiverse. Le 14 juillet, la Convention décide d'afficher dans toutes les municipalités le rapport de Chabot sur l'événement. Six jours plus tard, alarmé par la popularité de Corday, Fouquier-Tinville demande au Comité de Sûreté générale de diffuser ses lettres exactes (les journaux se fondaient sur des notes prises lors de leur lecture au procès), pour éviter toute ambiguïtée ${ }^{24}$. Or le même jour, une autre solution est choisie par le Conseil général de Paris, qui publie un violent contreportrait dans la Gazette de France nationale ${ }^{25}$. Deux jours plus tard, le Comité de Sûreté générale, finalement choqué de voir les lettres de Corday à la une des journaux, propose à Fouquier-Tinville une solution différente : la censure totale ${ }^{26}$. Il a donc fallu une semaine pour qu'une propagande officielle soit définie, et c'est bien long : les textes des lettres circulent déjà dans toute la France et au-delà, suscitant des vocations. Les conflits au sein du personnel politique et judiciaire expliquent en partie ces contradictions. Celui qui oppose Fouquier-Tinville et Montané après le procès est à ce titre intéressant. Le 29 juillet, ce dernier est destitué de la présidence du Tribunal révolutionnaire sur dénonciation de l'accusateur public, pour avoir rayé les mots «préméditation» et "contre-révolutionnaires» de la troisième question soumise aux jurés ${ }^{27}$. Sa défense montre que certains s'opposent à une répression trop dure, jusqu'à dénoncer les dérives despotiques de la justice d'exception ${ }^{28}$. Montané s'appuie en effet sur la loi : selon lui, le texte des questions aux jurés ne correspondait pas du tout aux renseignements obtenus lors des interrogatoires. Les enjeux politiques du procès sont forts : c'est Fouquier qui a écrit les questions à poser aux jurés, deux jours après avoir été convoqué par le ministre. Montané s'oppose à cette ingérence, qui court-circuite la délibération des jurés. En transformant la question «l'avait-elle fait avec des intentions contre-révolutionnaires, et avec préméditation", par "l'a-t-elle fait avec des intentions criminelles et préméditées d'assassiner sur la cime de la Montagne un représentant du peuple?", le juge prétend respecter scrupuleusement la procédure: "c'étaient là les expressions écrites de l'assassin", dit-il, en critiquant ouvertement l'arbitraire de Fouquier-Tinville. Pendant l'été 1793, la répression fait donc débat, ne correspond pas à un programme prédéfini, mais résulte de multiples luttes d'influences, dans lesquelles les stratégies personnelles prennent une certaine place.

Le procès permet aussi au gouvernement d'affirmer son autorité face aux sans-culottes, favorables à une radicalisation de la violence. Jacques Guilhaumou a montré le rôle des femmes, dont l'attachement à Marat les pousse à réclamer un nouveau supplice ${ }^{29}$. Le soir du 13 juillet, la rumeur de l'attentat se propage très vite, et quand on fait sortir Corday pour la conduire à l'Abbaye, des canons sont déjà placés aux deux extrémités de la rue des Cordeliers. Le moindre incident risque de mettre le feu aux poudres. Une foule immense bloque le passage, et il faut tout le charisme de Drouet pour persuader les 5 à 6000 personnes suivant la voiture, « au nom de la loi, de se retirer » et de ne pas la 
massacrer sur place ${ }^{30}$. Le lendemain, on rapporte que des groupes crient vengeance et menacent d'assassiner Marie Antoinette et le Dauphin. Craignant des émeutes incontrôlables, le Comité de Sûreté générale demande dans la nuit au commandant général de la force armée de Paris qu'il multiplie les patrouilles, redoublant sans le savoir une requête du Comité de Salut public du département ${ }^{31}$. Pour appeler la population au calme, on tire à 1200 exemplaires une adresse aux Parisiens ${ }^{32}$. Les autorités sanctionnent donc toute vengeance dépassant le juste châtiment : Legros, aide du bourreau, est ainsi emprisonné après avoir giflé la tête coupée de Corday ${ }^{33}$. L'attentat contre Marat réactive donc les débats des années 1791-1792 sur les crimes politiques (notamment consécutifs aux massacres de septembre), qui n'ont toujours pas abouti à un véritable consensus : certains (dont les héritiers de Marat) contestent encore à l'État le monopole du contrôle répressif, au nom de l'unité du peuple ${ }^{34}$.

Ce procès aurait aussi pu appuyer la répression de la révolte girondine du Calvados, mais il n'en est rien. Le 25 juillet, le très procédurier Montané adresse une copie du jugement au conseil municipal de Caen par formalité administrative, et non par propagande ${ }^{35}$. Les proches de Corday ne sont en outre presque pas inquiétés pour le meurtre de Marat, qui n'ajoute rien aux pressions que Jacques-François de Corday d'Armont subit depuis 1791 comme père d'émigré. La famille n'endure que quelques brimades ritualisées : quatre mois jour pour jour après le 13 juillet, le cortège de la société populaire de Caen, promenant un buste de Marat, s'arrête devant la maison de la tante qui logeait Corday, et entonne une chanson patriotique ${ }^{36}$. Ceci montre une volonté d'apaisement, permise par la débandade des troupes fédéralistes. L'existence de forts liens de solidarité entre autorités et populations explique aussi qu'on évite de mener à Caen une répression d'envergure et que les solutions locales soient préférées. Le renouvellement de Bonnet de Meautry (maire en 1789) comme représentant en mission témoigne du besoin de trouver un consensus en s'appuyant sur un passé fédérateur ${ }^{37}$.

11 Robert Allen a montré avec force que les tribunaux criminels départementaux ont clairement "rejeté les projets répressifs de l'État dans le domaine politique ${ }^{38}$ ". Le procès Corday montre quant à lui que même à Paris, au cœur des instances de répression, on est très loin d'une justice sans aspérités ni pitié. La riposte politique à la mort de Marat n'en est pas moins une réussite : son apothéose et le procès Corday ont détourné la demande populaire de vengeance dans le cadre de la loi et ont en même temps perdu les Girondins. Mais le procès, bâti sur une peur irrationnelle, ne se limite pas aux appareils administratifs et à leurs procédures, dont la logique s'apparente peut-être davantage à un « acte de foi $»^{39}$.

Une relecture culturelle

12 Le procès n'ayant pas fourni de véritable preuve juridique du complot, c'est le sens symbolique de l'exécution qui est en jeu : elle doit définitivement disqualifier « la fille Corday " aux yeux de tous et légitimer le pouvoir en place. Le 15 juillet, BillaudVarenne affirme à la Convention que l'existence du complot girondin est une question de croyance a priori, faute de pouvoir la prouver autrement: "Les traces matérielles manquent presque toujours; [...] il faut s'en tenir [...] à la simple conviction morale $»^{40}$. La façon dont l'exécution a été rapportée dans la presse a entraîné de nombreuses erreurs d'analyse dans une historiographie trop attachée aux discours : certains légitiment la répression en insistant sur le bon déroulement de l'exécution, d'autres insinuent le doute ou la critique en décrivant des anecdotes ayant perturbé la procédure. Le trajet 
de la charrette ouverte transportant Corday de la Conciergerie à la place de la Révolution témoigne d'une ritualisation (lenteur, parcours codifié) dont le dépouillement contraste avec le faste de la translation du corps-relique de Marat vers l'église des Cordeliers. Le spectacle de la mort publique doit servir une vengeance symbolique et régénérer l'assistance ${ }^{41}$. Mais l'acculturation prévue n'est pas toujours opératoire, et dans la foule, certains donnent l'impression de participer à des funérailles ante-mortem. Les représentations de Corday, promenée au milieu d'une foule hérissée de piques, sont très vite prisées des contre-révolutionnaires, qui recyclent toute une iconographie des martyres chrétiennes. La guerre des images n'est pas seulement religieuse : alors que le cortège désacralise le cérémonial du parcours aristocratique de la ville, la propagande royaliste transforme la charrette d'infamie ${ }^{42}$ en voiture de parade, restituant à la ci-devant Corday son prestige social : « Sur l'arrestation d'un bel Équipage devant une charrette/Cédez, nobles Coursiez, le pas à cette Rosse/Aujourd'hui, la charrette est plus que le carrosse ${ }^{43}$ ». La foule des exécutions révolutionnaires est donc aussi le lieu d'une certaine opposition politique : de nombreux témoignages montrent que les sympathisants de Corday s'y sont rendus en masse (notamment Adam Lux, député de Mayence), communiquant, se rencontrant peut-être.

Maints rituels de flétrissure visent à précipiter Charlotte Corday dans la mort ignoble : les injures, les mains liées, l'exposition mobile, la chemise rouge des assassins et l'allongement final, en imposant la contrition, prennent une valeur expiatoire. Les cheveux, coupés pour faciliter le passage de la lame, exhibent aussi sa déchéance sexuelle: cette mutilation est un rituel de long terme, infligé au XVIII ${ }^{e}$ siècle aux maquerelles et aux prostituées genevoises, que l'on retrouve jusqu'aux tonsures de la Libération, pour punir la collaboration horizontale ${ }^{44}$. Elle enferme Corday dans un genre monstrueux, et lui applique un châtiment corporel alors de plus en plus infligé aux meurtriers, mais aussi aux aristocrates et aux femmes sortant de leur rôle autorisé45. Au-delà, le rituel de l'exécution fait partie d'un système de croyances héritées de la tradition chrétienne: son bon déroulement est censé faire surgir la vérité, comme pendant les ordalies médiévales. Ainsi, la gifle de Legros est non seulement condamnée par les autorités, mais aussi désapprouvée par la foule comme barbarie inutile. Le député Sergent en livre un témoignage inédit. Le 18 juillet, il signale l'incident à Montané : «[...] Le peuple avait vu passer, avait conduit cette femme à l'échafaud sans insulter à ses derniers moments; il applaudissait intérieurement au jugement que lui réservait la peine due à son forfait, et plus son indignation était légitimement forte, plus son attitude, sa contenance tranquille le rendait fier et généreux. [...] Pourquoi le citoyen chargé de l'exécution de la loi s'est-il permis de le provoquer à des excès en ajoutant au supplice des outrages qu'on ne peut lui pardonner ? [...] Je demande au tribunal qu'il répare l'outrage fait à la nature, à la philosophie par l'un des exécuteurs qui conformément à la loi a montré au peuple la tête de la fille Corday, mais qui s'est permis de la couvrir de soufflets [...] $»^{46}$. On connaît les analyses de Michel Foucault sur les exécutions révolutionnaires: la guillotine est décrite comme un intermédiaire entre les supplices collectifs d'Ancien Régime et la mort d'État moderne ${ }^{47}$. Le témoignage de Sergent s'inscrit bien dans ce registre : la mise à mort légale doit satisfaire la demande populaire de vengeance et légitimer la puissance du pouvoir pour cimenter la paix civile. C'est pourquoi il loue la maturité politique des spectateurs, qui ne cèdent pas à l'impulsion vengeresse : façon calculée de contrer l'image contre-révolutionnaire d'un peuple mu par ses passions et incapable de résoudre les conflits politiques autrement que par la violence. C'est donc la capacité des révolutionnaires à installer l'État de droit qui est en jeu. Legros, en giflant la tête 
coupée, usurpe la prérogative étatique d'exercice de la violence, risque de flatter des instincts que le pouvoir ne pourrait canaliser. Pire encore : sa main, laissant sa trace sur les joues, rappelle trop la marque d'infamie d'Ancien Régime, et annule le rituel plus anonyme et égalisateur de la nouvelle économie de la mort légale.

Le procès Corday se situe donc à un moment charnière, où l'on cherche à ajuster les nouvelles institutions à la nation. D'où le respect scrupuleux de la liturgie et l'attente de signes légitimant la justice rendue. Plusieurs épisodes censés avoir eu lieu pendant l'exécution, rapportés ensuite tels quels, sont en fait à lire dans cette optique, car ils perturbent la fonction propitiatoire du rituel. À commencer par l'attitude de Corday, qui profite encore de cette mise en scène pour faire passer un certain nombre de messages, en affichant un calme qui a impressionné nombre de témoins. La condamnée ne joue donc pas le jeu des codes corporels attendus par le «bien mourir» des exécutions publiques, qui exige la soumission, et non l'abattement ou l'arrogance. Le pacte tacite, reposant sur un idéal chrétien faisant du corps le miroir de l'âme ${ }^{48}$, et liant les spectateurs au condamné, est rompu; le rituel risque d'être inopérant et même de se retourner contre ses destinataires. Chez certains le doute s'insinue sur la légitimité de la sentence : même la presse montagnarde reste un peu perplexe devant le courage serein de Corday ${ }^{49}$. Plusieurs légendes sont nées de cette disjonction, que l'historiographie n'a pas assez lues sous leur forme allégorique. La question de la beauté de Charlotte Corday, souvent mise en avant, en fait partie. Ces jugements de goût, encore trop pris pour des témoignages fiables d'une "beauté réelle ", sont en fait liés au spectacle d'un corps triomphant contre toute attente, alors même que celui de Marat s'était décomposé aux yeux de tous, nécessitant l'abrègement de son apothéose. Autre exemple : dès le soir du 13 juillet, on murmure dans les assemblées de section qu'une épidémie de peste se répand dans Paris ${ }^{50}$; avec Corday l'aristocrate, c'est tout l'Ancien Régime qui revient, suivi par son cortège de peurs collectives liées aux châtiments divins. De même, un orage aurait éclaté lors de l'exécution ${ }^{51}$. Outre la référence biblique explicite, il procède de la sacralité des signes atmosphériques accompagnant les événements historiques. Au Moyen Age, la pluie ou la foudre annonçaient la protection ou le châtiment de Dieu. L'orage du 17 juillet n'est pas une pure invention : il a bien plu ce jour-là, mais au nord de Paris, et non sur la place de la Révolution. Il ne faut donc pas voir dans cette légende une volonté consciente de falsifier l'histoire, mais bien plutôt la nécessité de trouver un sens à la Révolution : les producteurs de ces légendes sont eux-mêmes pris dans un système de représentations dont ils dépendent inconsciemment. Les brochures aussitôt publiées et les journaux, reprennent ici le ton des occasionnels des siècles précédents, consacrés aux événements transgressant les lois naturelles.

Tirée des premiers récits de l'exécution, cette aquarelle rappelle l'iconographie des premières martyres chrétiennes. Corday, main sur le cœur, éclairée par la lumière divine au centre de la composition, est entourée par une foule sombre et hostile ${ }^{52}$.

Un des thèmes récurrents de l'historiographie est l'autopsie qui aurait été pratiquée sur le corps décapité de Corday pour vérifier sa virginité ${ }^{53}$. Or aucune source ne la mentionne. Ce type de légende est en fait un topique de l'hagiographie féminine du Moyen Age, appliqué aux abbesses et moniales injustement accusées de dépravation ${ }^{54}$. Cet épisode a donc vraisemblablement été imaginé dans les milieux contrerévolutionnaires pour comparer Corday aux saintes martyres. La participation de David à l'autopsie, et l'origine de la rumeur (son atelier), renforcent le parallèle entre 
l'apothéose de Marat et le procès Corday: le peintre, ordonnateur de la pompe de Marat, incarne l'oracle de l'histoire, celui qui la donne à voir et qui l'interprète, lisant comme les haruspices dans les entrailles de la morte le futur d'une imprévisible Révolution. Cet épisode renvoie aussi à l'examen médico-légal effectué sur le corps de Marat, au centre de son martyrologe. L'invention de ce rituel morbide a donc bien pour effet de concurrencer la mémoire de l'Ami du Peuple: Antoine de Baecque a montré avec l'exemple de Mirabeau l'importance croissante des autopsies, puis de l'exposition des cadavres dont la description médicale et la vue doivent authentifier la pureté ou au contraire sanctionner la dépravation contre-révolutionnaire ${ }^{55}$. Les anecdotes relatives aux cheveux jouent le même rôle : leur coupe, le don d'une mèche-relique au peintre Hauer, et les anecdotes plus triviales (Charlotte Corday était-elle une vraie blonde ?) montrent que la féminité de Corday imprègne les combats de mémoire en $1793^{56}$. Le débat suscité dès la fin du XVIII ${ }^{e}$ siècle par sa décollation, repris cent ans plus tard par le criminologue Cesare Lombroso, est également le reflet d'une incertitude sur l'issue de la Révolution. Certains auraient en effet aperçu les joues de Corday rougir une fois la tête coupée ; cette légende, propagée dans les milieux modérés ${ }^{57}$, pose une nouvelle fois le problème de la légitimité de l'exécution. Elle place Corday dans un genre hagiographique qui s'appuie sur toute une tradition de grands récits (motif de la pendue miraculée ${ }^{58}$ ) connus sous la Révolution pour comparer le rituel d'exécution au martyre des modèles de la foi ${ }^{59}$. Les estampes en clair-obscur représentant Corday (mais aussi Madame Roland, Marie-Antoinette...) écrivant à son père dans son cachot ( ( Pardonnez-moi mon cher Papa... »), reprennent le thème iconographique de la femme repentie, associé à Marie-Madeleine. Ces vanités montrent que les catholiques ont parfois critiqué Corday ; mais en inventant son ultime repentir, ils légitiment aussi son intégration dans leurs valeurs spirituelles ${ }^{60}$. Ces scènes de cachot élégiaques témoignent aussi du rôle que joue déjà Corday dans l'éveil d'une sensibilité romantique. Les brochures bon marché, les journaux et les estampes qui diffusent ses lettres ou ses paroles les plus marquantes, reprennent souvent le registre expiatoire des complaintes, vendues lors des exécutions publiques et proposant le vrai-faux testament du condamné, ainsi que ses derniers mots ${ }^{61}$. D'autres gravures du XIX siècle inspirées de l'iconographie chrétienne montrent Corday le doigt pointé vers le ciel, indiquant l'origine de sa foi et de sa force. Les formes de la guillotine, fondées sur des règles géométriques intangibles ${ }^{62}$, rappellent facilement celles de la croix. La comparaison avec les martyres chrétiennes a certes ses limites: la représentation de Corday en buste, pourvue de ses attributs (chapeau, lettre et couteau), évoque trop le meurtre, peu compatible avec les principes de la foi ${ }^{63}$. Ces images très réversibles sont d'ailleurs souvent reprises par les anti-Corday, à toutes les époques. Mais l'extrait de La Chaste Suzanne, pièce jouée à Paris en 1793, inspirée d'une scène de l'Ancien Testament, témoigne de la fusion des images entre culture chrétienne et Révolution. Suzanne est injustement accusée par deux vieillards lubriques, Accaron et Barzabas, de les avoir séduits en prenant son bain. La scène se passe sur une place publique où elle doit être jugée : Suzanne monte sur l'estrade.

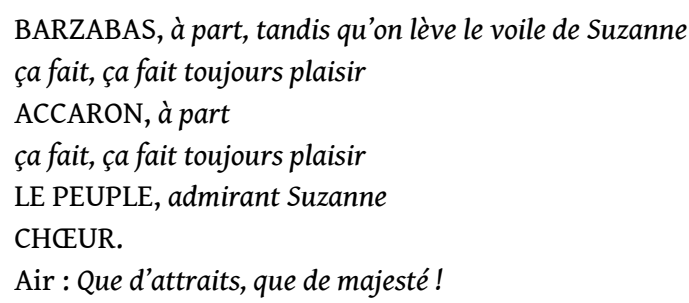


Que d'attraits, que d'aménité!

Que de grâces, que de beauté!

(Accaron et Barzabas restent comme pétrifiés à

l'aspect de Suzanne ${ }^{64}$ )

Suzanne (comme Charlotte, également comparée à d'autres femmes de l'Ancien Testament) oppose au regard impudique des traitres sa pureté physique et morale. Le dévoilement de son visage préfigure alors celui de la vérité. Dernier trait commun à ces anecdotes : elles portent toutes sur une critique de la féminité du corps de Corday. Les discours sexistes en témoignent, ainsi que les mutilations et viols symboliques fantasmés ou pratiqués sur elle (mains baladeuses, toilette, exhibition, dénudation des épaules et couchage du corps avant la décollation...). Ces châtiments visent à exposer le corps traitre des femmes et à le détruire symboliquement. D'autres en ont fait les frais : deux mois auparavant, Théroigne de Méricourt avait été fessée aux portes de la Convention. L'enlaidissement de Charlotte Corday procède de cette même logique : il vise à dénoncer son impureté morale et disqualifier l'action des femmes dans la Révolution. Toute cette propagande relève d'un arrière-plan culturel de très long terme ${ }^{65}$, mais elle s'inscrit aussi dans le contexte particulier d'une " période virile » de la Révolution : le 20 juillet, Olympe de Gouges est arrêtée, et trois mois plus tard, les clubs féminins sont interdits.

Les autorités ne peuvent tout contrôler. Lorsque le journaliste des Annales de la République française croit avoir vu Corday placer elle-même sa tête sous l'échafaud, et quand il se souvient que "la bascule a semblé tomber plus lentement que de coutume ", il reconnaît que tout ne s'est pas passé comme prévu ${ }^{66}$.

Une relecture biographique

L'attentat, trop lu comme un fait divers, devait en réalité être perpétré au beau milieu de la Convention nationale. Corday affiche plusieurs fois sa volonté initiale de marquer l'opinion par un assassinat spectaculaire au cœur de la République ${ }^{67}$. Le choix de la victime n'est pas non plus aléatoire. Il vise à la fois à satisfaire les milieux modérés et à choquer les radicaux, en rééditant, contre Marat cette fois, l'attentat contre Lepeletier de Saint-Fargeau, poignardé au Palais Royal le 20 janvier de la même année. Mais contrairement à Paris, qu'elle cite, elle s'assure d'être identifiée. Pendant tout son procès, elle s'attache à justifier l'attentat et à lui donner le maximum de publicité. Consciente de la censure qui va lui être opposée, elle la contourne en exploitant tous les moyens d'expression possibles: les interrogatoires, les lettres écrites en prison, le procès mais aussi l'exécution sont ainsi utilisés pour infléchir le cours de l'histoire en faveur de sa cause et de son image. Le premier interrogatoire mené après l'attentat, terminé après minuit en présence de plusieurs députés et d'administrateurs de police, montre qu'elle prend des précautions ${ }^{68}$. Les réponses de Corday sont claires, précises et ne portent le plus souvent que sur les questions posées. Sa préoccupation est à cet instant de protéger son entourage en insistant sur son isolement, que l'historiographie croira à la lettre. Sans avoir encore de vraie stratégie (elle pensait être aussitôt mise en pièces: l'image des sans-culottes parisiens est alors déplorable dans les milieux girondins), elle se met déjà en scène en affirmant que "son projet n'était pas un projet ordinaire " et insiste sur sa détermination en parlant à plusieurs reprises de son "dessein ${ }^{69}$ ». Aspirant à devenir l'icône d'une certaine esthétique révolutionnaire, elle met en exergue son propre sacrifice pour la patrie.

Dès le lendemain, elle travaille activement pour sa postérité : elle demande notamment au Comité de Sûreté générale qu'un portrait d'elle puisse être fait pour laisser un 
souvenir à ses proches. Une telle tentative, vouée à l'échec, ne prouve en rien une quelconque naïveté. Elle se rattache tout d'abord à la tradition aristocratique des portraits familiaux. Mais quand elle argue que les autorités pourront aussi s'en servir comme repoussoir pour les futures générations, elle va bien plus loin. Non seulement elle ironise sur la propagande appliquée contre elle, mais elle la subvertit, jouant de l'ambiguïté de ce type d'image, suscitant à la fois l'aversion et l'intérêt. Elle connait la tradition des portraits de criminels et de guillotinés visant à montrer par leur apparence physique qu'ils sont des monstres sociaux ${ }^{70}$; elle sait aussi leur popularité au sein des classes populaires. Ce double jeu vise à concurrencer la mémoire des martyrs de la Révolution et le culte naissant de Marat, qu'elle désacralise en disant que «si le département met sa figure vis a vis celle de St Fargeau il pourra faire graver ses paroles en lettres d'or ${ }^{71}$ ». L'image de Corday comme criminelle-née, attisant la curiosité de la presse populaire et de l'école française d'anthropologie criminelle dès la fin du XIX ${ }^{\mathrm{e}}$ siècle est donc liée à une volonté qu'elle eut elle-même d'utiliser ce registre. La demande rejetée, elle facilite la réalisation du portrait le plus fidèle, réalisé par JeanJaques Hauer. Mené par la rumeur chez Marat juste après l'assassinat, ce garde national décide d'assister au procès et de dessiner Corday, qui, le remarquant, se serait tournée vers lui. Le peintre a ensuite revendiqué une connivence réciproque. Le tableau en porte l'empreinte, dans la tradition physiognomonique : les traits y sont censés refléter l'âme du modèle. Cette théorie formulée par Lavater a été confortée par les travaux de Cuvier et de Darwin au XIX ${ }^{\mathrm{e}}$ siècle, et cautionne les analyses criminologiques dans la recherche des origines biologiques des déviances sociales. Chez Hauer, qui manie le savant code des signes corporels auquel on était alors habitué, Corday n'apparait guère comme une dégénérée : la blondeur et les yeux clairs suggèrent la pureté, le nez et le menton allongés affirment la détermination, le front dégagé signale l'intelligence, la blancheur témoigne de la noblesse. C'est une vestale que l'on contemple, vêtue de blanc immaculé, les mains pieusement posées sur les genoux ${ }^{72}$. Ce tableau, vite gravé par Anselin, jouera dans les milieux modérés et contre-révolutionnaires, le rôle inverse de celui de David, mais avec trois mois d'avance : celui d'icône commémorative que l'on se passera sous le manteau ou que l'on accrochera aux côtés d'autres figures emblématiques. Inversement, beaucoup d'autres portraits font de Corday une criminelle-née : on la représente alors petite et trapue, rousse, les traits grossiers, peu avenants, les arcades sourcilières prononcées, le front étroit, affublée du châle rouge des assassins ${ }^{73}$. Mais la propagande anti-Corday ne s'est que très peu attardée sur son cas, étant donné les risques de détournement que de telles images pouvaient présenter.

21 La vraie fausse Lettre à Barbaroux est un autre moyen trouvé par Corday pour détourner la censure. Écrite en prison, habituellement crue comme une confession sincère révélant son inconséquence et sa légèreté d'esprit, elle procède en réalité d'une véritable stratégie. Jouant sur les représentations sociales dominantes qui font $\mathrm{du}$ genre épistolaire un apanage féminin, Corday prétexte une correspondance privée pour faire passer sans risque un certain nombre de messages politiques. L'historiographie a parfois été déroutée par l'aspect bric-à-brac de la lettre, qui commence par le récit picaresque de son voyage à Paris, ponctué par les vaines tentatives de séduction d'un compagnon de route. Ce passage révèle la culture aristocratique de Corday, qui excelle dans l'art très codifié de la conversation de salon et le recyclage de scènes empruntées à la littérature galante (scène de sérénade sous une fenêtre). Il lui permet aussi de tromper ses censeurs et de leur faire croire à la dimension privée de la missive. Mais il lui fournit aussi l'occasion de se positionner comme femme (c'est sur toute une culture 
virile qu'elle ironise) et comme révolutionnaire modérée (ses compagnons de route montagnards sont ridiculisés). Enfin, cet épisode en apparence sans intérêt traduit une certaine distanciation ironique vis-à-vis de son "épopée ». Le récit de sa semaine héroïque, contaminé au XIX ${ }^{e}$ siècle via la littérature d'édification par la structure narrative et les fonctions symboliques du conte (Le Petit Chaperon Rouge, La Belle et la Bête $)^{74}$, est donc aussi élaboré par Corday elle-même. Dans le reste de la lettre, elle développe son idéologie complexe, proche de celle des Girondins, issue d'une contrerévolution modérée liée à son entourage familial, mais qui a rejoint la révolte girondine par culture du compromis et du légalisme. Plusieurs fois, Corday se présente comme le fer de lance des troupes fédéralistes en marche vers Paris. Le 13 juillet correspond en effet à l'offensive partie de Caen; par inexpérience, elle n'en devine peut-être pas la faiblesse, et elle n'imagine pas les condamner en assassinant Marat. La question de la naïveté de l'attentat, souvent avancée pour disqualifier Corday comme objet historique, nous paraît téléologique: elle procède d'un jugement a posteriori et souligne la persistance d'une vision positiviste d'un progrès consciemment orienté par les actions de grands hommes, écartant les "perdants » et outsiders. Manifestement, les conséquences de l'attentat ont complètement échappé à Corday. Elles n'en sont pas moins importantes et intéressantes pour l'historien, dont l'analyse est faussée si, comme le critique littéraire, il ne dépasse pas le principe d'intentionnalité des acteurs/ auteurs de l'histoire, qui efface les mécanismes de réception et d'appropriation, mais aussi la part d'imprévu de tout événement. Il ne s'agit pas de réhabiliter Corday, mais bien de reconstituer la complexité du changement historique et de sa mise en récit. La stratégie de justification de Corday l'a parfois desservie. Ainsi, en citant Thomas Corneille (1625-1709) $)^{75}$ dans la lettre qu'elle écrit à son père ("C'est le crime qui fait la honte, et non pas l'échafaud»), elle ne s'imagine pas donner naissance à un débat acharné : Corday descend-elle de Pierre Corneille, et si oui est-ce en ligne directe ? La filiation, maintes fois prouvée, a souvent été utilisée pour démontrer sa prédestination au tragique, voire au meurtre : depuis la fin du XVIII ${ }^{e}$ siècle, l'influence des thèses de l'hérédité des caractères acquis sur l'analyse historique, fait de l'ascendance familiale de Corday un élément central de sa biographie. Faire table rase de ces croyances est tentant, mais conduit à une autre erreur : nier la stratégie de l'honneur, déployée par Corday pendant son procès. Car la citation nous donne une clef de lecture irremplaçable pour comprendre l'attentat (presque un duel) qui, envisagé au sein d'une éthique aristocratique ${ }^{76}$, ne déshonore pas la famille. Corday était-elle illuminée? Le style des lettres est, il est vrai, haché, et les traits de plus en plus vigoureux trahissent une excitation croissante. Mais l'argumentaire se révèle aussi fourni et référencé : Corday est consciente des contradictions qu'impliquent le meurtre d'un représentant du peuple, quand on prétend défendre la légalité et la paix civile. Pour se justifier, elle revendique un thème récurrent dans la littérature politique depuis le XVII ${ }^{e}$ siècle : le tyrannicide. Selon elle, le pouvoir de Marat étant illégitime, celui-ci peut être tué sans compromettre l'État de droit. Des références connues étayent sa démonstration : elle se compare à Brutus et, quelques mois plus tôt, citait Raynal et Milton ${ }^{77}$. Néanmoins, l'argumentation se noie dans des digressions plus superficielles et une croyance que le meurtre de Marat sonne l'avènement de la paix. Il y a du quichottisme chez Corday. Mais il s'inscrit dans une esthétique du sublime imprégnant tous les discours de l'époque, et il ne faut pas la prendre au premier degré. Les réponses données à Montané révèlent d'autre part une indéniable culture politique: elle y défend les girondins révoltés en démontrant leur attachement à l'unité de la République, place son 
engagement dans la désapprobation des massacres de septembre et des coups d'état du 31 mai et du 2 juin, affiche son indépendance politique et son intérêt pour la presse révolutionnaire de tous bords. Contrairement au premier interrogatoire, mené dans le feu de l'action, celui-ci prend l'aspect d'une joute verbale que Corday a eu le temps de préparer. C'est encore plus vrai lors du procès, mené en public: elle y protège son entourage, justifie son action, et trouve un vrai sens de la formule (" j'ai tué un homme pour en sauver cent mille »). Corday n'est pas Lacenaire, elle ne cherche pas à ridiculiser l'institution judiciaire ${ }^{78}$, dont elle augmente au contraire la gravité, pour la détourner à son profit . Lors de l'exécution, son calme, son maintien et sa jeunesse tranchent avec la putréfaction nauséabonde du corps affaissé de Marat, déambulant sur un lit de parade dans une ambiance dramatique. Corday, éduquée chez les bénédictines, détourne ce qui devait être sa déchéance, en adoptant une posture sacrificielle : elle sera exaltée par l'iconographie chrétienne du XIX ${ }^{e}$ siècle qui représentera son corps en gloire sur le modèle de l'Assomption.

22 Auteur de sa propre mémoire, Corday échoue cependant à faire diffuser l'Adresse aux Français, son véritable testament politique écrit la veille de l'attentat, et très vite censuré. Mais son principal échec tient dès 1793 dans l'impossibilité des milieux modérés à cautionner le meurtre de Marat, représentant du peuple. L'attentat «terroriste» de Corday, même s'il n'est pas utilisé à charge lors du procès des girondins à l'automne, contredit leur culture de l'éthique politique, de la légalité et de l'État de droit. Si certains chantent son héroïsme (Salles, Louvet, Pétion, Chénier), beaucoup d'autres bottent en touche (Barbaroux, Gorsas) ou la critiquent (Madame Roland). Corday, en poignardant un député, empêche elle-même la création d'une contre-mémoire d'envergure en transgressant le consensus minimal de l'État de droit et en sapant un des fondements de la Révolution : la représentation nationale.

Les approximations des institutions, les contradictions politiques et la fébrilité des sensibilités, dévoilent d'abord l'incertitude face à une histoire qui s'accélère. Ce qui n'empêche pas une certaine efficacité : l'attentat est récupéré par les Montagnards pour disqualifier les Girondins, canaliser les plus radicaux, et classer le problème du fédéralisme normand. Politiquement, le 13 juillet 1793 répond à des questions sur la violence restées ouvertes depuis septembre 1792 : les modérés se satisfont que Marat, bouc-émissaire des massacres, soit enfin puni. D'autre part, l'exécution de Corday réconcilie la répression d'État et la violence populaire: l'apothéose de Marat et la déchéance de Corday ont été vécues comme un même ensemble de rituels inversés interrogeant la violence et sa résolution politique. À ce sujet, on s'aperçoit combien l'économie moderne de l'exécution capitale est loin d'être achevée en 1793, taraudée par la tentation des anciens supplices (Corday est bien traitée en régicide, mais comment prendre acte du transfert de sacralité du roi à la nation, et lui trouver un châtiment approprié ?) et détournée à leur profit par les individus qui savent s'insinuer dans les failles d'un système inabouti. Les rituels de l'exécution ont été différemment lus par les acteurs de la Révolution; mais tous y ont vu le même sacrifice fondateur et ont puisé aux mêmes sources culturelles pour défendre leur version de l'histoire. De multiples traditions narratives très codifiées se sont insinuées dans l'historiographie, dont il faut retracer la généalogie, pour ne pas aplanir l'épaisseur du récit parvenu jusqu'à nous. La mise en évidence de récits superposés et imbriqués a ainsi permis de dégager l'évolution des enjeux soulevés par l'attentat, à la fin du XVIII ${ }^{e}$ siècle et après. L'archive dessine enfin les contours de Corday, outsider de l'histoire et personnagefrontière de la Révolution. Très identifiable par les catégories politiques et sociales de 
l'historien, elle cumule néanmoins une série de "handicaps historiographiques " (crime politique, sexe féminin, image contre-révolutionnaire, etc...) qui expliquent son éviction de l'histoire majoritaire et sa captation par des mouvements plus sulfureux (extrême-droite, mouvements terroristes, régionalisme), brouillant encore un peu plus la compréhension de sa trajectoire qui n'a été jugée acceptable que lorsqu'elle était cantonnée à la trivialité du fait divers.

\section{NOTES}

1.Jacques Guilhaumou, La mort de Marat, Paris, Complexe, 1989, 169 p., Jean-Claude BONNET (dir), La mort de Marat, Paris, Flammarion, 1989, 510 p., Sophie WAHNICH, La liberté ou la mort, essai sur la Terreur et le terrorisme, Paris, La Fabrique, 2003, 111 p.

2.Christian DELPORTE, Annie DUPRAT (dir.), L'Événement, images, représentation, mémoire, Paris, Créaphis, 2003, 265 p.

3.Dernier avatar: Martial DEBRIFFE, Charlotte Corday, Paris, France-Empire, 2005, 268 p.

4.Haïm BURSTIN, «En guise de conclusion : quelques remarques historiographiques », dans Jean-Clément MARTIN (dir.), La Révolution à l'œuvre. Perspectives actuelles dans l'histoire de la Révolution française, Rennes, PUR, 2005, 375 p.

5.Jean-Clément MARTIN, Révolution et Contre-Révolution en France. Les rouages de l'histoire, Rennes, PUR, 1996, 230 p., p. 11.

6.Blandine BARRET-KRIEGEL, « Histoire et politique, ou l'histoire, science des effets ", AESC, n6, 1973, p. 1437-1462.

7.Carlo GINZBURG, Le juge et l'historien, considérations en marge du procès Sofri, Paris, Verdier (1ère éd. 1991), 1997, 187 p., p.107-108 ; de même pour le procès Carrier : Corinne GOMEZLE CHEVANTON, Carrier et la Révolution Française en 30 Questions, La Crèche, Geste Editions, $63 \mathrm{p}$.

8.Moniteur Universel, 15 juillet 1793.

9.AN BB 16703.

10.AN W 277.

11.BM Versailles, f. Vatel F 677.

12.BM Versailles, f. Vatel, $\mathrm{F} 675$.

13.AN W 277, AN BB 16703

14.AN W 277 et AN BB 16 703, p. 8 et 9.

15.AN W 277.

16.Bulletin du Tribunal Criminel Révolutionnaire, ${ }^{\circ}$ LXXI.

17.AP, séance du 14 juillet 1793.

18.AN W 277.

19.Charles VATEL, Procès criminel de Charlotte Corday devant le Tribunal Révolutionnaire, Paris, Poulet-Malassis, 1861, p.VIII.

20.Bulletin du Tribunal Révolutionnaire, LXXII.

21.Archives Parlementaires, Convention nationale, séance du 19 juillet 1793.

22.BM Versailles, f.Vatel, $\mathrm{F} 675$. 
23.Archives Parlementaires, Convention nationale, séance du 16 juillet 1793.

24.BM Versailles, f. Vatel, F 677.

25. Gazette de France Nationale, 20 juillet 1793.

26.AN W277.

27.AN W277.

28.BHVP, m. 603041, fol 37.

29.Jacques GUILHAUMOU, op.cit, p. 30.

30.Lettre de Guiot à la société populaire de Semur, 15 juillet 1793, BM Versailles, f. Vatel, m. F 675.

31.BM Versailles, f. Vatel, m. F676.

32. AN BB 381.

33.Journal de Perlet, 22 juillet 1793, Scrutateur Universel, 21 juillet 1793.

34.Eric DE MARI, La mise hors de la loi sous la Révolution française (19 mars 1793-9 thermidor an II), thèse de doctorat en droit, université de Montpellier I, 1991, 678 p., p. 37.

35.BM Versailles, f. Vatel, m. F 679.

36.Affiches, Annonces et Avis divers, ou Journal du département du Calvados, 25 brumaire an II.

37.Jean-Clément MARTIN, Contre-Révolution, Révolution et Nation en France, 1789-1799, Paris, Seuil, 1998, 367 p. et Michel BIARD, Missionnaires de la République. Les représentants du peuple en mission (1793-1795), Paris, CTHS, 2002, 623 p.

38. Robert ALLEN, Les tribunaux criminels sous la Révolution et l'empire, 1792-1811, Paris, PUR, 2005, 318 p, p. 272.

39.Ainsi parle Carlo Ginzburg de la rhétorique du verdict du procès Sofri, op.cit., p. 149. 40.AP, 15 juillet 1793. Ce nouveau type de " preuve » avait déjà été proposé par Carnot en 1791 pour accélérer la répression : Eric DE MARI, op.cit, p. 36.

41.Sur la fonction régénérante des exécutions publiques : Michel BEE, « Le spectacle de l'exécution dans la France de l'Ancien Régime », Annales ESC, 38e année, 1983/ $\mathrm{N}^{\circ} 4$, p. 843-850.

42.Valérie ROUSSEAU-LAGARDE, Daniel ARASSE, La guillotine dans la Révolution, Institut Français de Florence, 1987, 181 p., p. 88.

43.BM Versailles, f. Vatel m.F676.

44.Michel PORRET, Le crime et ses circonstances. De l'esprit de l'arbitraire au siècle des Lumières selon les réquisitoires des procureurs généraux de Genève, Genève, Droz, 1995, 562 p., p. 362, et Fabrice VIRGILI, La France « virile ». Des femmes tondues à la Libération, Paris, Payot, 2000, $421 \mathrm{p}$.

45.Antoine DE BAECQUE, Le corps de l'Histoire, métaphores et politique (1770-1800), Paris, Calmann-Lévy, 1993, 435 p.

46.BM Versailles, f. Vatel F 675.

47.Michel foucAult, Surveiller et Punir, Paris, Gallimard, 1975, p. 22.

48.Laurence GUIGNARD, « Les supplices publics au XIX ${ }^{\mathrm{e}}$ siècle. L'abstraction du corps », dans Michel PORRET (dir.), Le corps violenté, du geste à la parole, Paris, Droz, 1998, p. 173.

49.Jacques GUILHAUMOU, op.cit., p. 72.

50.Journal de Perlet, 15 juillet 1793.

51.Jacques GUILHAUMOU, ibidem, p. 72-73.

52.Aquarelle anonyme, 0,150/0,705, début XIX ${ }^{\mathrm{e}}$ siècle, musée Lambinet, Versailles, inv. 907. Surl'iconographie des martyres chrétiennes : Martine VASSELIN, « Entre horreur du supplice et vision béatifique: regards sur le martyre dans la peinture italienne et 
française à l'époque moderne ", dans RégisBERTRAND, Anne CAROL, L'Exécution capitale, une mort donnée en spectacle, $\mathrm{XVI}^{e}$-XXe siècle, Aix, PUP, 2003,p. 203-236.

53.BONNEVILLE, Almanach des Gens de Bien, an III.

54.Jacques LE GOFF, Saint Louis, Paris, Gallimard, 1996, p. 105.

55.Antoine DE BAECQUE, La gloire et l'effroi, sept morts sous la Terreur, Paris, Grasset, 1997.

56.Nina R.GERBALT, «The Blonding of Charlotte Corday », Eighteen Century Studies, n³8, 2004, p. 207-221

57.Daniel ARASSE, La guillotine et l'imaginaire de la Terreur, Paris, Flammarion., p. 51 et suiv.

58.Roger CHARTIER (éd), Les usages de l'imprimé, Paris, Fayard, 1987, p. 83-129.

59.Michel BEE, art.cit.

60.« Marie-Anne Charlotte de Corday d'Armans, âgée de 26 ans, écrivant sa dernière lettre à son père ", gravure à l'aquatinte, $0,163 / 0,294$, anonyme, à Paris, chez Basset, 1793. On retrouve des connotations expiatoires voisines dans les images funèbres de Napoléon : Emmanuel FUREIX, « La mort de Napoléon. Images et cristallisations de l'événement (1821-1831) », dans Christian DELPORTE, Annie DUPRAT, op.cit,p. 168.

61.Michel BEE, art. cit., p. 848.

62.Daniel ARASSE, op. cit., p. 73.

63. "Charlotte Corday, assassin de Marat », gravure en couleurs anonyme, 105/.085, Musée Lambinet, Versailles, inv.892.

64.La Chaste Suzanne, pièce en deux actes, Théâtre du Vaudeville, 5 janvier 1793, Paris, Maret, 1793, II, 3, BM Versailles, f. Vatel m. F676 p. 12.

65.Natalie Z. DAVIs, Les cultures du peuple. Rituels, savoirs et résistances au XVIe s., Paris, Aubier, 1979, $444 \mathrm{p}$.

66.Jacques GUILHAUMOU, op.cit., p. 73.

67.Bulletin du Tribunal Criminel Révolutionnaire, $\mathrm{n}^{\circ}$ LXXI, AN W 277.

68.AN F7 4407 d.1.

69.AN W 277.

70.Le fait divers, Paris, RMN, 1982, Daniel ARASSE, op.cit.

71.AN W 277.

72.Jean-Jacques HAUER (1751-1829), Marie-Anne-Charlotte de Corday dite Charlotte Corday (1768-1793), huile sur toile, 0,60/0,47, inv. MV 4615, Musée National de Versailles.

73. « Charlotte Corday », gravure en couleur $(0,363 / 0,235)$, BM Rouen, Est. Az. Hedou, D.V. 5362 .

74.Catherine Velay-VALLANTIN, « Le miroir des contes », dans Roger CHARTIER (éd), op. cit.,p. 128-185. Il y a beaucoup à étudier sur l'influence du conte dans l'historiographie révolutionnaire. Voir CAMPION-VINCENT Véronique, SHOJAEI KAWAN Christine, « Marie-Antoinette et son célèbre dire : deux scénographies et deux siècles de désordres, trois niveaux de communication et trois modes accusatoires ", AHRF, $\mathrm{n}^{\circ}$ 327, janvier-mars 2002, p. 29-56.

75.Le frère de Pierre Corneille (1606-1684).

76.Osons parler d'une « culture du fer » pour Corday : Pascal BRIOIST, Hervé DREVILLON, Pierre SERNA, Croiser le fer, Violence et culture de l'épée dans la France moderne (XVI ${ }^{-}$-XVIII ${ }^{e}$ siècle), Paris, Champ Vallon, 2002, $514 \mathrm{p}$.

77.AD Calvados, $2 \mathrm{~L} 146$.

78.Anne-Emmanuelle DEMARTINI, L'Affaire Lacenaire, Paris, Aubier, 2001, 430 p. 


\section{RÉSUMÉS}

Rituel inverse de l'apothéose de Marat, le procès Corday obéit à plusieurs logiques. Dans un contexte de crise, il doit d'abord fournir une réponse rassurante à la demande de vengeance des sans-culottes: il est donc organisé comme une véritable arme idéologique visant le complot contre-révolutionnaire. S'il permet d'expérimenter des pratiques institutionnelles plus efficaces pour faire appliquer des mesures exceptionnelles, il témoigne surtout de l'improvisation et des divisions des autorités politiques et judiciaires. Également vécu par l'opinion comme un rituel de régénération collective, il mobilise tout un système de croyances héritées qui ont donné naissance à des légendes tenaces dans l'historiographie. Il révèle enfin la personnalité complexe de Charlotte Corday, qui tente d'en faire sa propre apothéose.

The Trial of Corday: a return to the sources. The opposite ritual of the apotheosis of Marat, the trial of Corday served different purposes. In the context of a crisis, it was intended to supply a reassuring response to the demand for vengeance expressed by the sans culottes: it was , therefore, organized as a veritable ideological weapon against the counterrevolutionary plot. If it permitted the experimentation of more efficient institutional practices for the use of exceptional measures, it revealed no less the divisions and improvisations among the judicial and political authorities. In addition, the trial was perceived as a ritual of collective regeneration; it mobilized an entire system of inherited beliefs that gave birth to tenacious historiographic legends. It revealed as well the complex personality of Charlotte Corday, who tried to transform the trial into an exercise in self-aggrandizement.

\section{INDEX}

Mots-clés : Contre-Révolution, justice, violence, Marat, Corday, guillotine

\section{AUTEUR}

\section{GUILLAUME MAZEAU}

Doctorant, Université Paris I Panthéon-Sorbonne

Institut d'histoire de la Révolution française

17 rue de la Sorbonne

75005 Paris

mazeau.guillaume@free.fr 